\title{
Metformin in Combination with Malvidin Prevents Progression of Non-Alcoholic Fatty Liver Disease via Improving Lipid and Glucose Metabolisms, and Inhibiting Inflammation in Type 2 Diabetes Rats
}

\author{
Wenlan Zou ${ }^{1}{ }^{1 *}$ \\ Chen Zhang ${ }^{1} *$ \\ Xuefang Gu (iD ${ }^{2}$ \\ Xiaohong $\mathrm{Li}^{3}$ \\ Huiming $\mathrm{Zhu}^{4}$ \\ 'Department of Endocrinology, Fifth \\ People's Hospital of Suzhou, Suzhou, \\ Jiangsu, 215100, People's Republic of \\ China; ${ }^{2}$ Department of Endocrinology, \\ Xiangcheng District Second People's \\ Hospital of Suzhou, Suzhou, Jiangsu, \\ 215100, People's Republic of China; \\ ${ }^{3}$ Department of Liver Disease, Fifth \\ People's Hospital of Suzhou, Suzhou, \\ Jiangsu, 215100, People's Republic of \\ China; ${ }^{4}$ Department of Gastroenterology, \\ Fifth People's Hospital of Suzhou, Suzhou, \\ Jiangsu, 215100, People's Republic of \\ China
}

*These authors contributed equally to this work
Correspondence: Wenlan Zou Department of Endocrinology, Fifth People's Hospital of Suzhou, No. 10 Guangqian Road, Xiangcheng District, Suzhou City, Jiangsu, 215100, People's Republic of China

Email zhouw12102@I63.com
Background: Non-alcoholic fatty liver disease (NAFLD) is one of the primary causes of chronic liver disease and is closely linked to insulin resistance, type 2 diabetes mellitus (T2DM), and dyslipidemia. However, no effective drug therapies have been approved to treat this disease. The present research aimed to evaluate the therapeutic effects of the combination of oral hypoglycemic drug metformin (MET) and a natural product malvidin (MAL) on hepatic damage in HFD/STZ-induced diabetic rats.

Methods: Sprague-Dawley rats were divided into five groups: normal control group (NC), diabetic control group (DC), DC+MET group, DC+MAL group, and DC+MET+MAL group and treated for eight weeks. Blood and liver tissue samples were collected for metabolic parameters, histological, and RT-qPCR analysis.

Results: Our findings indicated that hyperglycemia, insulin resistance, hyperlipidemia, and non-alcoholic fatty liver disease (NAFLD) in diabetic rats were alleviated after oral treatment with MET and MAL, particularly their combination therapy. Besides, the expression of SREBP-1c, ACC, FAS, IL-6, IL-8, and NF- $\kappa$ B mRNA was down-regulated by MET + MAL, and the expression of PPAR $\alpha$, CPT1, and LPL was up-regulated by MET+MAL.

Conclusion: The evidence of this research indicated that the combination therapy may represent an efficient strategy against NAFLD in T2DM rats via improving lipid and glucose metabolisms, and inhibiting inflammation.

Keywords: non-alcoholic fatty liver disease, diabetes mellitus, combination, lipogenesis, malvidin, inflammation

\section{Introduction}

Type 2 diabetes mellitus (T2DM) is a chronic metabolic disorder that is characterized by persistent hyperglycemia due to the impaired islet cell function and is considered a serious global health problem. ${ }^{1,2}$ T2DM causes not only high blood glucose, but also non-alcoholic fatty liver disease (NAFLD), diabetic nephropathy, and dyslipidemia. ${ }^{3}$ NAFLD is defined by excessive deposition of fat in hepatocytes, which is closely linked to several metabolic diseases, including insulin resistance, T2DM, and dyslipidemia. ${ }^{4,5}$ Additionally, a previous report indicated that the prevalence of NAFLD in conjunction with diabetes and obesity. T2DM may be a major risk factor for the progression from steatosis to steatohepatitis, even cirrhosis. ${ }^{6}$ Furthermore, NAFLD is rapidly becoming the primary cause of end- 
stage liver disease and hepatocellular carcinoma. ${ }^{7}$ Thus, in long-term treatment, alleviating the complications of T2DM is just as important as lowering blood glucose levels. A growing number of the report indicated that persistent hyperglycemia affects the inflammatory mechanisms of the liver, which in turn promotes liver damage in aspects of liver fibrosis and lipid accumulation. $^{8-10}$ Additionally, previous reports have demonstrated that anti-inflammatory compounds could alleviate liver damage, control blood glucose, and improve insulin resistance. ${ }^{11,12}$ Thus, therapeutic suppression of hepatic inflammation may be an effective treatment for T2DM and its metabolic syndrome. ${ }^{13,14}$

Metformin (MET) is the most prescribed oral antidiabetic drug and derived from plant Galega officinalis. ${ }^{15}$ It has been reported that MET could decrease hepatic lipid levels in T2DM patients; ${ }^{16}$ MET also inhibited the inflammatory response in non-alcoholic steatohepatitis via STAT3-mediated autophagy induction. ${ }^{17}$ Despite the health benefits of MET, previous studies have reported several side effects of MET, including diarrhea, lactic acidosis, and gastrointestinal disorders. ${ }^{18}$ Therefore, researchers have proposed the combination therapy to eliminate the side effects of oral anti-diabetic drugs and also to enhance its effectiveness, such as MET combined with other herbal agents. ${ }^{19,20}$

Recent researches on natural products, especially anthocyanins, have focused on preventing NAFLD and T2DM. For example, the anthocyanins extract from black soybean seed coat was shown to hypolipidemic and hypoglycemic effects in $\mathrm{T} 2 \mathrm{DM} ;{ }^{21}$ it has been reported that an anthocyaninrich berry could improve insulin sensitivity in obese and overweight populations; ${ }^{22}$ bilberry anthocyanins were shown to alleviate western diet-induced NAFLD via improving gut microbiome dysbiosis and hyperlipidemia. ${ }^{23}$ Malvidin (MAL) is an anthocyanins compound belonging to a subgroup of flavonoids, is highly present in berries, flowers, and red grape skin. It has been extensively investigated due to its anti-inflammatory, anti-tumor, and cardioprotective effects. ${ }^{24-26}$ Additionally, MAL could protect endothelial cells via suppressing peroxynitrite-induced NF- $\mathrm{KB}$ activation; ${ }^{27} \mathrm{MAL}$ was shown to inhibit hepatocellular carcinoma via regulating metastasis, proliferation, and apoptosis. ${ }^{28}$ However, there were no reports on the effects of MET combined with MAL on NAFLD. Therefore, the present study aimed to explore the therapeutic effects of MET or MAL alone or in combination on hyperglycemia, insulin resistance, hyperlipidemia, and NAFLD in diabetic rats, and we hypothesized that MET in combination with MAL could alleviate NAFLD via improving lipid and glucose metabolisms, and suppressing inflammation.

\section{Materials and Methods}

\section{Animals and Treatments}

Male Sprague-Dawley rats (6-8 weeks old, 180-220 g) were procured from the Model Animal Research Center of Nanjing University (Nanjing, China). All rats were housed in an experimental animal center of Suzhou Fifth People's Hospital at $50 \pm 5 \%$ humidity and $20 \pm 2^{\circ} \mathrm{C}$ under a $12 / 12$ $\mathrm{h}$ light/dark cycle with a freely available diet and water. The protocol involving animals was approved by the Ethics of Animals Experiments Committee of Suzhou Fifth People's Hospital (approval number: 2019011B) and performed following the National Standard (GB/T 35892-2018) for Laboratory Animals Care and Use.

After seven days of acclimation, except for the normal control group, other group rats were administrated with a high-fat diet (HFD) for 12 weeks. HFD consisting of $0.5 \%$ sodium cholate, $3 \%$ cholesterol, $10 \%$ lard, $20 \%$ sucrose, and $66.5 \%$ standard chow diet. After six weeks of HFD, DC, DC+MET, DC+MAL, and DC + MET + MAL groups were fasted overnight and intraperitoneally injected with a single dose of STZ ( $35 \mathrm{mg} / \mathrm{kg}$ body weight) in freshly citrate buffer, while $\mathrm{NC}$ group rats were intraperitoneally injected with an equivalent volume of citrate buffer. After one week of STZ injection, the fasting blood glucose levels were assayed, and glucose levels $>11.1 \mathrm{mmol} / \mathrm{L}$ were considered diabetic. The animals were randomly divided into five groups ( $\mathrm{n}=10$ per group). Normal control group (NC): rats received standard chow diet neither drug treatment nor diabetes induction; diabetic control group (DC): rats received $\mathrm{HFD} / \mathrm{STZ}$ and no drug administration; MET protection group (DC+MET): rats received $\mathrm{HFD} / \mathrm{STZ}$ and administrated with MET (100 mg/kg body weight); MAL protection group (DC+MAL): rats received $\mathrm{HFD} / \mathrm{STZ}$ and administrated with MAL (100 mg/kg body weight); MET + MAL protection group (DC+MET+MAL): rats received HFD/STZ and administrated with the combination of MET (100 mg/kg body weight) and MAL (100 mg/kg body weight). From week 0 to week 12, the MET+MAL or MET or MAL or vehicle (DMSO) was treated to rats by oral gavage daily. The dosage of MET or MAL used was based on previous reports. ${ }^{20,25}$ The body weight of rats was recorded throughout the experiment. The detailed experimental scheme is shown in Figure 1A. At the end of the 
experiment, all rats were anesthetized with pentobarbital (30 $\mathrm{mg} / \mathrm{kg}$ body weight) after fasting overnight. Then, the blood sample was collected from inferior vena cava and centrifuged at $4^{\circ} \mathrm{C}$ for $15 \mathrm{~min}$ at $3500 \times \mathrm{g}$, and the supernatant was collected and stored at $-20^{\circ} \mathrm{C}$ for further analysis. The liver tissues were quickly collected and washed with physiological saline, and weighted. The liver tissue samples were fixed in $10 \%$ paraformaldehyde solution for histopathological assessment or stored at $-20^{\circ} \mathrm{C}$ for further analysis.

\section{Oral Glucose Tolerance Test (OGTT) and Insulin Tolerance Tests (IPITT)}

One day before the termination of the experiment, the rats fasted overnight. The rats were received glucose (2 $\mathrm{g} / \mathrm{kg}$ body weight) by oral gavage for OGTT. The rats were injected intraperitoneally with insulin (2 units $/ \mathrm{kg}$ body weight) for IPITT. Blood samples were obtained from the tail vein over the time course of 0 to $120 \mathrm{~min}$ and the blood glucose levels were measured using commercial kits.

\section{Serum Biochemical Analysis}

The levels of interleukin 6 (IL-6), IL-8, blood glucose, insulin, leptin, triglyceride (TG), total cholesterol (TC), low-density lipoprotein cholesterol (LDL-C), highdensity lipoprotein cholesterol (HDL-C), aspartate aminotransferase (AST), and alanine aminotransferase (ALT) in serum were measured using commercial kits (Jiancheng Bioengineering Institute, China) based on the manufacturer's protocol. Homeostasis model assessment of insulin resistance (HOMA-IR) was calculated as follows: (fasting blood glucose $\times$ serum insulin)/22.5. The item number of the corresponding kits was as follows: glucose (A154-1-1), insulin (H203-1-1), leptin (H174), TG (A110-1-1), TC (A111-1-1), LDL-C (A113-1-1), HDL-C (A112-1-1), AST (C010-2-1), and ALT (C009-2-1).

\section{Determination of Hepatic Glucose and Lipid Metabolism Markers and Inflammation Parameters}

The liver tissues were homogenated in ice physiological saline and centrifuged at $5000 \times \mathrm{g}$ for $15 \mathrm{~min}$, and the supernatant was collected. The commercial protein kit was used to measure the total protein content of the supernatant based on the manufacturer's protocol
(Jiancheng Bioengineering Institute, China). The levels of phosphoenolpyruvate carboxykinase (PEPCK), glucose 6-phosphatase (G6Pase), glycogen synthase (GS), TC, TG, IL-6, IL-8, and NF-kB in liver tissues were measured using commercial kits (Jiancheng Bioengineering Institute, China) based on the manufacturer's protocol. The item number of the corresponding kits was as follows: PEPCK (A131-1-1), G6Pase (H5801), GS (H388-1), IL-6 (H007), IL-8 (H008), and NF-אB (H202).

\section{Histopathology}

The liver tissues were washed with physiological saline and then fixed with polyformaldehyde (4\%) for 24 $\mathrm{h}$. Then, the tissue samples were embedded in paraffin and cut into slices with a thickness of $4 \mu \mathrm{m}$. These sections were deparaffinized in xylene and rehydrated with a series of concentrations of ethanol. After washing with physiological saline, these sections were incubated in Oil Red $\mathrm{O}$ reagent (Jiancheng Bioengineering Institute, China) based on the manufacturer's protocol. Additionally, the liver sections were examined by hematoxylin and eosin staining (H\&E). The stained sections were observed under a light microscope (Olympus, Tokyo, Japan).

\section{Quantitative Real-Time PCR}

Total RNA of liver tissues was extracted using the Trizol reagent (Invitrogen, Carlsbad, CA, USA). $2 \mu \mathrm{g}$ of extracted RNA was reverse transcribed into cDNA by the Prime Script RT reagent Kit (Takara, Shiga, Japan). Then, quantitative real-time PCR was carried out using the SYBR Green Master Mix (Thermo Fisher, USA) on an Applied Biosystems StepOnePlus System (Thermo Fisher, USA). The primer sequences used for measuring gene expression were shown in Supplementary file (Table 1S). The qPCR was carried out in duplicate, and the gene expression was reported as $2^{-\Delta \Delta \mathrm{Ct}}$. The GAPDH is used as the internal control.

\section{Statistical Analysis}

The data were presented as mean $\pm \mathrm{SD}$ and analyzed using the GraphPad Prism 6.0 software (San Diego, CA, USA). Statistical differences among multiple groups were evaluated by one-way analysis of variance (ANOVA), followed by Dunnett's Multiple Comparison Test. It was considered statistically significant when $P<0.05$. 


\section{Results}

\section{General Effects of Combined Drug} Therapy on HFD/STZ-T2DM Model Rats

The diabetic rats exhibited typical diabetic symptoms, including emaciation, polyphagia, and polydipsia. We observed these diabetic symptoms in the DC group. The body weight of rats in HFD groups increased continuously during the first 6 weeks of the experiment. However, the body weight of rats in the HFD/STZ group declined obviously after injection of STZ (Figure 1B). The final body weight of HFD/STZ rats was lower than that of NC rats (Figure 1C), while the food consumption and water consumption of HFD/STZ rats were higher than that of NC rats (Figure $1 \mathrm{~F}$ and $\mathrm{G}$ ). The combination of MET and MAL alleviates these diabetic symptoms in the DC group $(P<$ 0.01). Besides, the liver weight and liver index of HFD/STZ rats were higher than that of NC rats (Figure 1D and E). The combination of MET and MAL decreased the liver weight, and liver index of rats compared to the DC group $(P<0.01)$.

\section{Combined Drug Therapy Alleviated Insulin Resistance}

As shown in Figure 2, treatment with MET or MAL alone obviously decreased glucose, and insulin intolerance (AUC) compared with the DC group $(P<0.001)$. Interestingly, the reduction in the $\mathrm{DC}+\mathrm{MET}+\mathrm{MAL}$ was higher than that of the MET or MAL alone.

Besides, insulin resistance was evoked in the DC group as displayed by hyperglycemia, hyperinsulinemia, and increased HOMA-IR as compared to the NC group (Figure $3 \mathrm{~A}-\mathrm{C}$ ). Treatment with MET or MAL alone decreased fasting blood glucose level, serum insulin level, and HOMA-IR compared with the DC group. Interestingly, the decline in the DC+MET+MAL was higher than that of the MET or MAL alone. These findings indicated that the combined drug therapy alleviated glucose and insulin intolerance in T2DM model rats.

\section{Combined Drug Therapy Ameliorated HFD/STZ-Induced Adverse Changes in}

\section{Serum Biochemical Markers}

As shown in Figure 3D-G, HFD/STZ resulted in an obvious increase in the serum levels of leptin, TC, TG, and LDL-C compared to those in the NC group. These parameters were reduced in T2DM rats administered with MET or MAL alone. Importantly, the reduction in the DC+MET+MAL was higher than that of the MET or MAL alone. HFD/STZ also resulted in a significant decrease in the serum levels of HDL-C compared to that in the NC group (Figure 3H). The HDL-C levels were increased in T2DM rats administered with MET or MAL alone. Importantly, the combination of MET and MAL caused an increase in the serum levels of HDL-C as compared to MET or MAL alone treated groups.

\section{Combination of MET and MAL Alleviated NAFLD in HFD/STZ-Induced T2DM Rats}

The liver function indexes, including serum ALT and AST, were observed to be increased in the DC group (Figure 4A

\section{A}
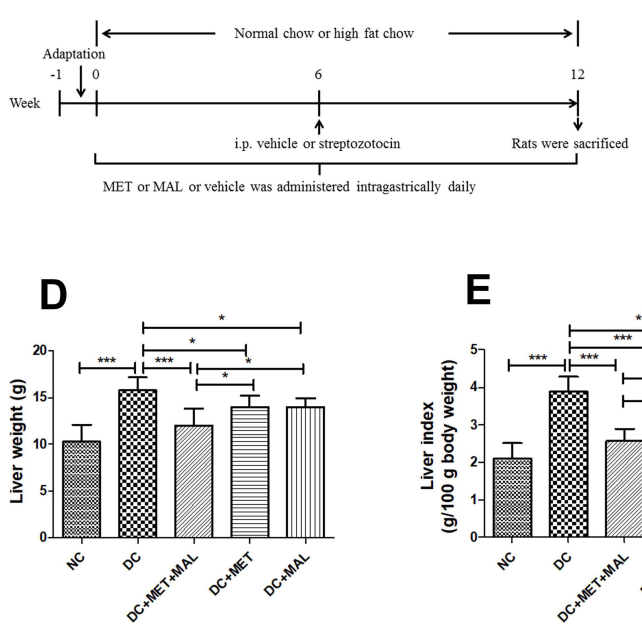

E

\section{B}
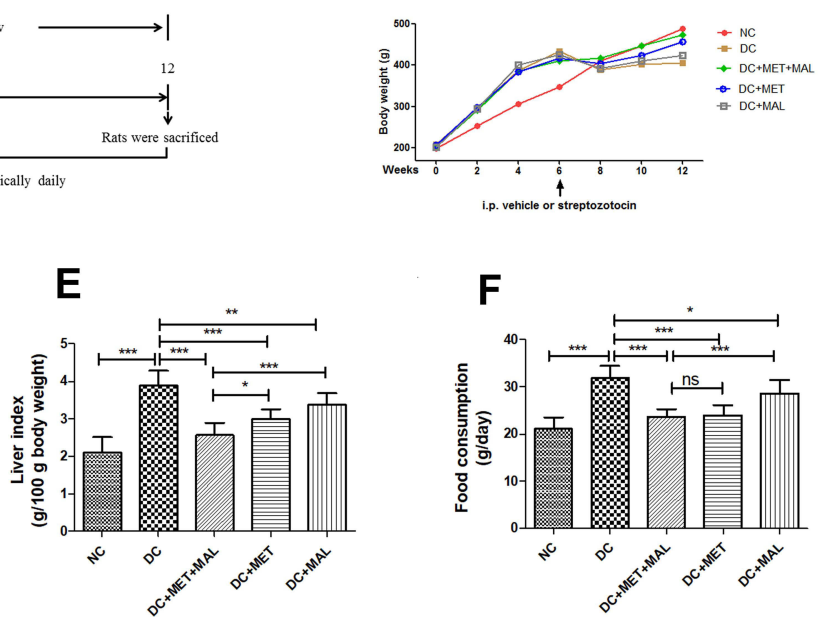

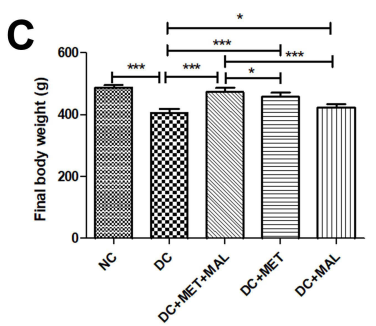

G

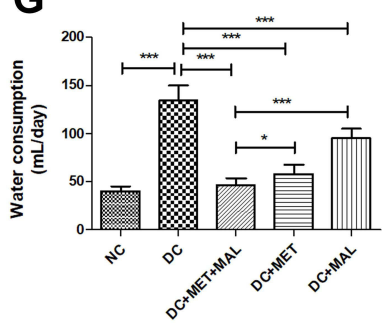

Figure I Effects of MET, MAL, and MET+MAL on T2DM related characteristics in HFD/STZ-T2DM model rats. (A) The detailed experimental scheme; (B) Body weight changes during the experiment; (C) Final body weight; (D) Liver weight; (E) Liver index; (F) Food consumption; (G) Water consumption. 

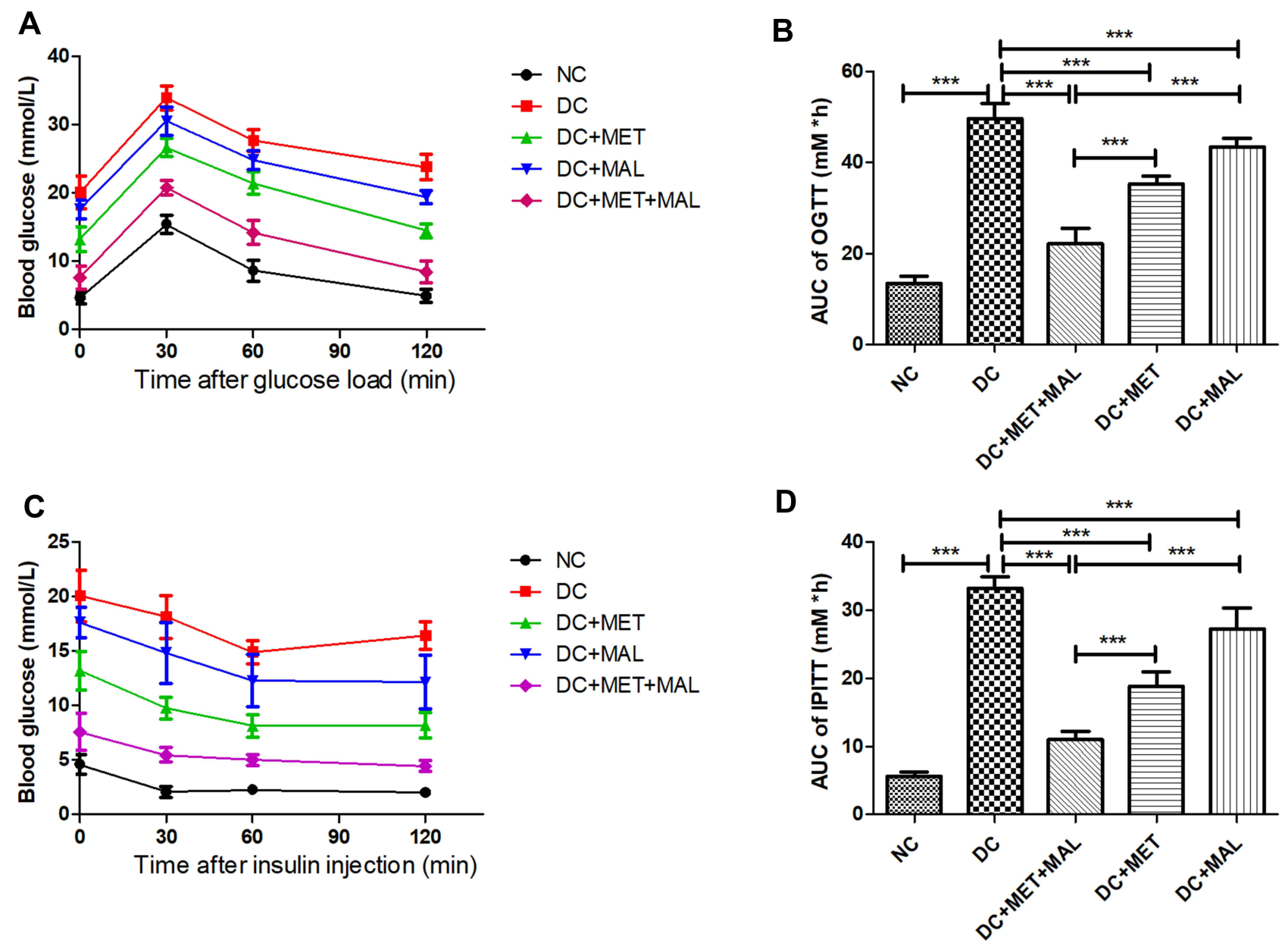

Figure 2 Effects of MET, MAL, and MET+MAL on insulin sensitivity in HFD/STZ-T2DM model rats. (A and B) Oral glucose tolerance test (OGTT); (C and D). Insulin tolerance tests (IPITT). The data were presented as mean \pm SD, $(n=8$ for all groups). $* * * P<0.00 I$.

and $\mathrm{B})$. These liver function indexes were declined in T2DM rats administered with MET or MAL alone. Importantly, the combination of MET and MAL led to a decrease in the serum levels of ALT and AST as compared to MET or MAL alone treated groups. Besides, H\&E staining showed regular cell morphology, uniform cytoplasm, and clear liver lobules structure in the $\mathrm{NC}$ group under a light microscope. In the DC group, hepatocytes were swollen and disordered arrangement; lipid vacuoles were widely distributed in the hepatocytes; there was obvious infiltration of inflammatory cells. MET, MAL, and combination of MET and MAL all alleviated these liver pathological changes in T2DM rats, and the effect on the combined group was greater (Figure 4F). Hepatic levels of TC and TG were increased by HFD/STZ and declined by treatment with MET or MAL alone. Interestingly, the decline in the $\mathrm{DC}+\mathrm{MET}+\mathrm{MAL}$ was higher than that of the MET or MAL alone (Figure $4 \mathrm{C}$ and D). The Oil red $\mathrm{O}$ staining was performed to further evaluate the effects of combined drug therapy on hepatic lipid accumulation (Figure 4E). MET, MAL, and combination of MET and MAL all decreased hepatic neutral lipids in T2DM rats, and the effect on the combined group was greater.

\section{The Combination of MET and MAL Inhibited the Expression of Hepatic Genes Related to Lipogenesis}

As shown in Figure $5 \mathrm{~A}-\mathrm{C}$, the expression of genes involved in lipogenesis (SREBP-1c, ACC, and FAS) were measured by RT-qPCR to further investigate the underlying mechanisms of combined drug therapy regulated hepatic fat accumulation on HFD/STZ-induced T2DM rats. The expression of SREBP-1c, ACC, and FAS was higher in the hepatic tissues of the DC group compared with the NC group. Treatment with MET or 

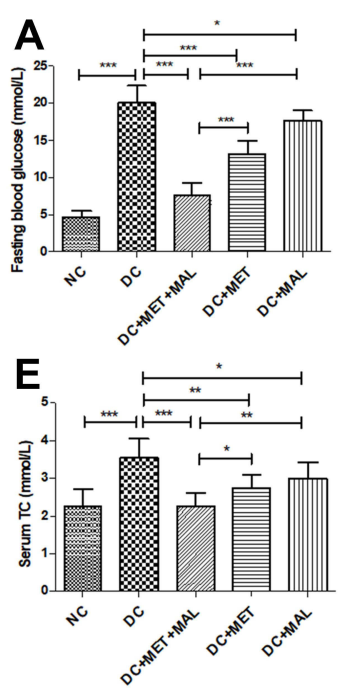

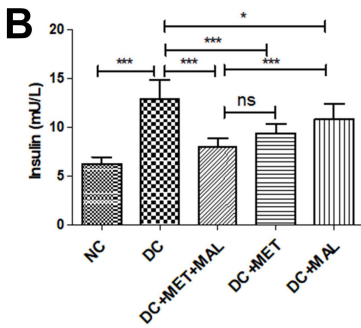

$\mathbf{F}$

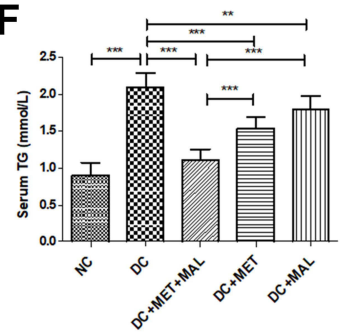

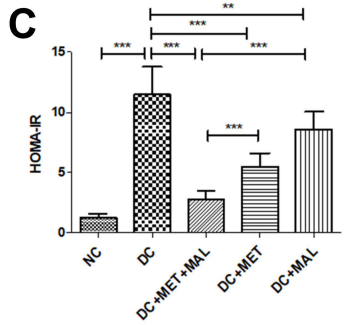

G

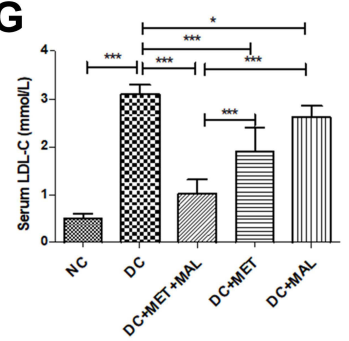

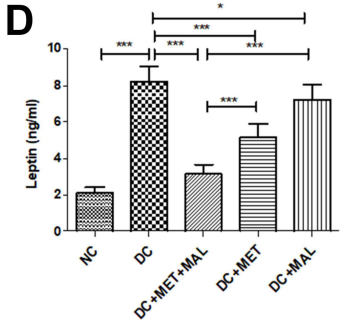

H

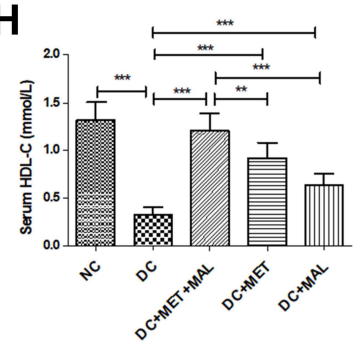

Figure 3 Effects of MET, MAL, and MET+MAL on serum biochemical parameters in HFD/STZ-T2DM model rats. Insulin resistance relative marker, including fasting blood glucose (A), insulin (B), HOMA-IR (C), and leptin (D). Lipid metabolism parameters, including serum TC (E), serum TG (F), serum LDL-C (G), and serum HDL-C (H). The data were presented as mean $\pm S D$, ( $n=8$ for all groups). ***P<0.00I;** $<<0.01$; $* P<0.05$.

A

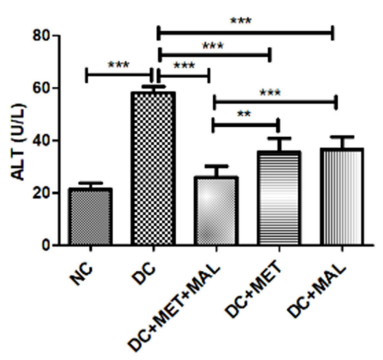

NC

E

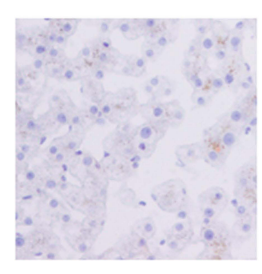

$\mathbf{F}$

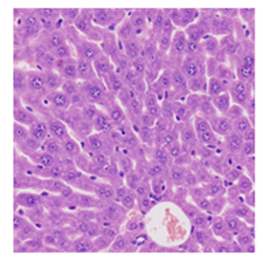

B

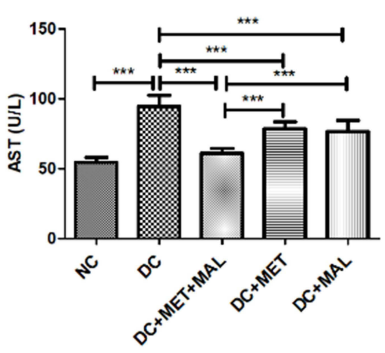

DC
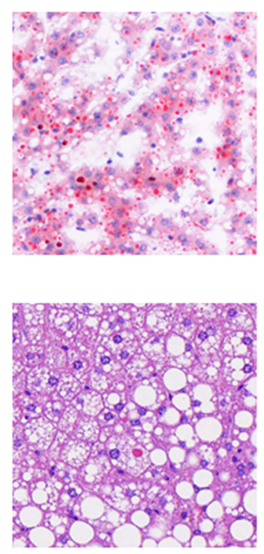

C

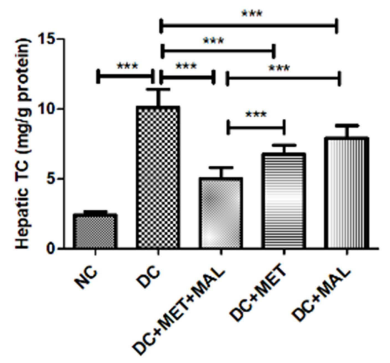

DC+MET+MAL $\quad$ DC+MET
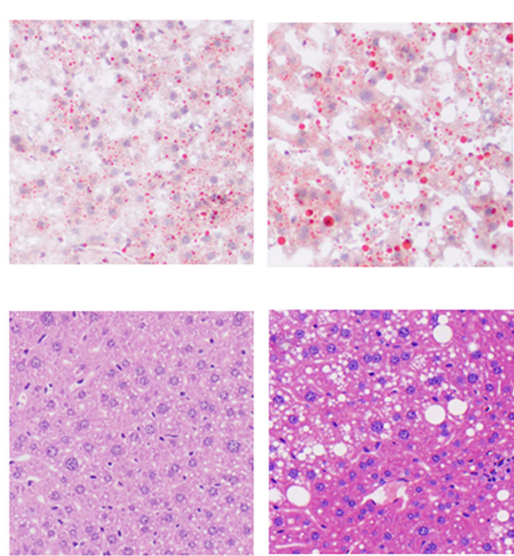

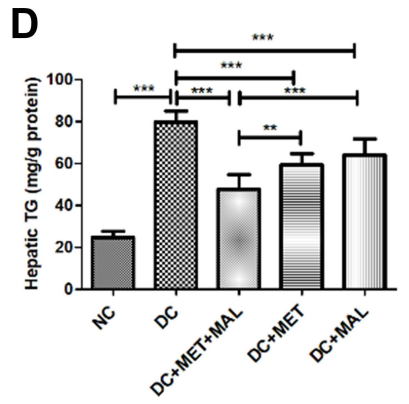

DC+MAL
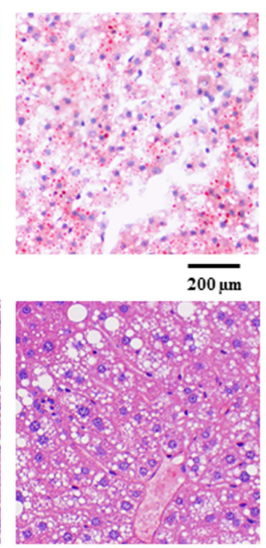

$\overline{100 \mu \mathrm{m}}$

Figure 4 Effects of MET, MAL, and MET+MAL on NAFLD in HFD/STZ-T2DM model rats. (A) The activity of ALT in serum of rats; (B). The activity of AST in serum of rats; (C). Hepatic levels of TC; (D). Hepatic levels of TG; (E). Lipid accumulation in the hepatic tissue of rats was evaluated by Oil red $O$ staining, the scale bar is $200 \mu$ m; $(\mathbf{F})$. Representative photos of H\&E staining from liver tissue, the scale bar is $100 \mu \mathrm{m}$. The data were presented as mean $\pm S D$, ( $\mathrm{n}=8$ for all groups). $* * * P<0.00 \mathrm{I}$; $* * P<0.01$. 

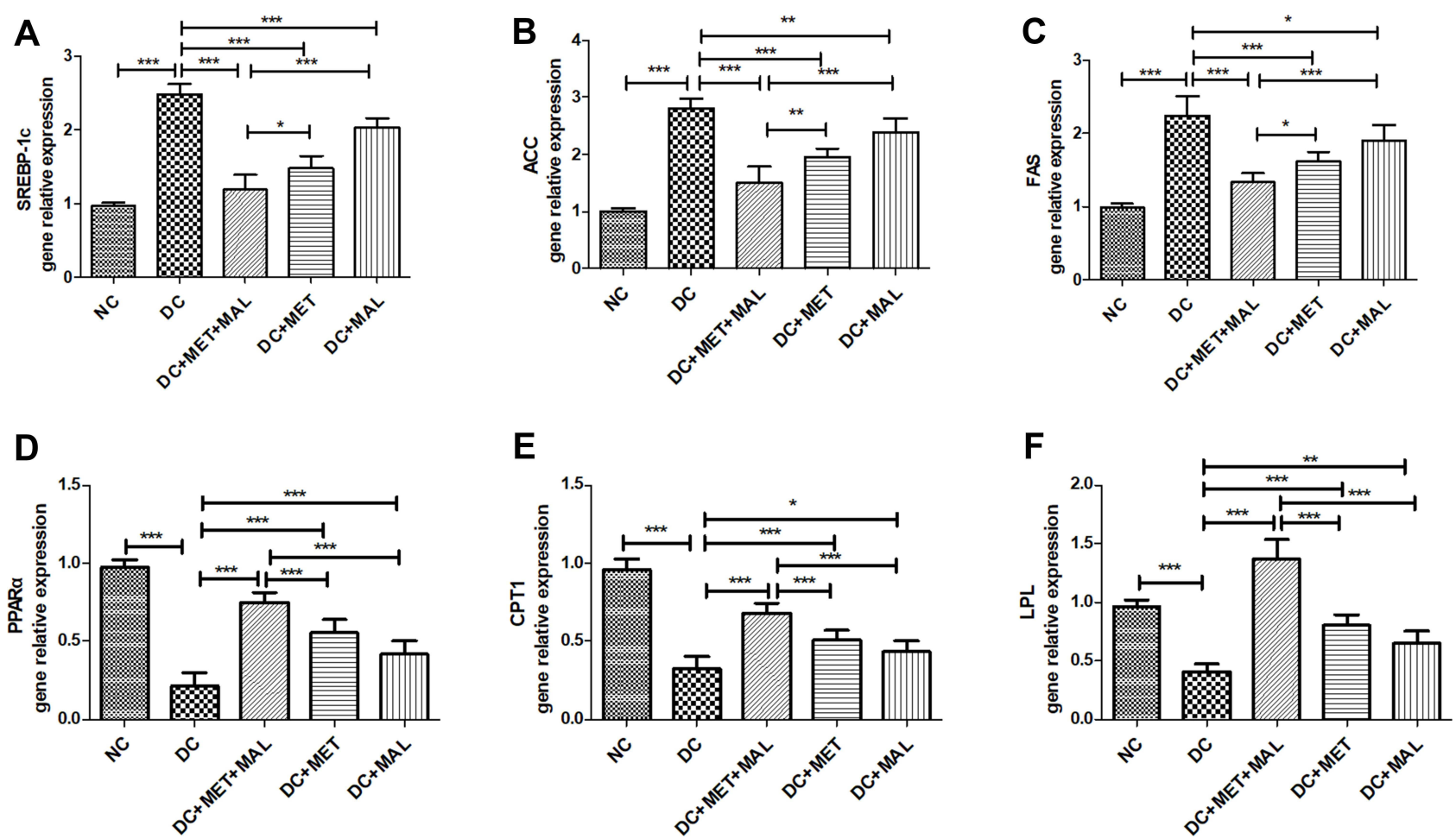

Figure 5 Effect of combined drug therapy on hepatic gene expression involved in lipid homeostasis. The relative expression of hepatic genes involved in lipogenesis, including SREBP-IC (A), ACC (B), and FAS (C). The relative expression of hepatic genes involved in fatty acid oxidation, including PPAR $\alpha$ (D), and CPTI (E). The relative expression of hepatic genes involved in lipid metabolism, including LPL (F). The data were presented as mean $\pm S D$, $(n=6$ for all groups). $* * * P<0.00$ I; $* * P<0.0$ I; $* P<0.05$.

MAL alone significantly down-regulated these hepatic genes involved in lipogenesis. Interestingly, the downregulation in the $\mathrm{DC}+\mathrm{MET}+\mathrm{MAL}$ was higher than that of the MET or MAL alone.

\section{Combination of MET and MAL Up-Regulated Expression of Hepatic Genes Involved in Fatty Acid Oxidation and Lipid Metabolism}

As shown in Figure 5D-F, the gene expression involved in fatty acid oxidation (PPAR $\alpha$ and CPT1) and lipid metabolism (LPL) were down-regulated by HFD/STZ and upregulated by treatment with MET or MAL alone. Interestingly, the upregulation in the $\mathrm{DC}+\mathrm{MET}+\mathrm{MAL}$ was higher than that of the MET or MAL alone.

\section{The Combination of MET and MAL Relieves Hepatic Inflammation Induced by HFD/STZ}

The serum and hepatic levels of IL-6, IL-8, and NF-kB were increased in the DC group compared to the NC group (Figures 6 and 7). Treatment with MET or MAL alone decreased IL-6, IL-8, and NF- $\kappa$ B levels induced by HFD/ STZ. Interestingly, the reduction in the DC+MET+MAL was higher than that of the MET or MAL alone. Besides, the gene expression of IL-6, IL-8, and NF- $\mathrm{BB}$ in hepatic tissues was measured by RT-qPCR also to further demonstrate that hepatic inflammation caused by HFD/STZ was inhibited by the combination of MET and MAL (Figure 8). These results indicated the potency of the combination of MET and MAL in inhibited hepatic inflammation in HFD/ STZ-induced T2DM rats.

\section{Effect of Combined Drug Therapy on the Activities of Enzymes Related to Glucose Metabolism}

The activities of glycogen synthesis- and gluconeogenesisrelated enzymes were measured to explore the effects of combined drug therapy on hepatic glucose metabolism. As shown in Figure 9, the activities of G6Pase and PEPCK in the DC group were significantly higher than those in the NC group. Treatment with MET or MAL alone decreased G6Pase, and PEPCK activities induced by HFD/STZ. Interestingly, the decrease in the $\mathrm{DC}+\mathrm{MET}+\mathrm{MAL}$ was higher than that of the MET or MAL alone. Besides, the 

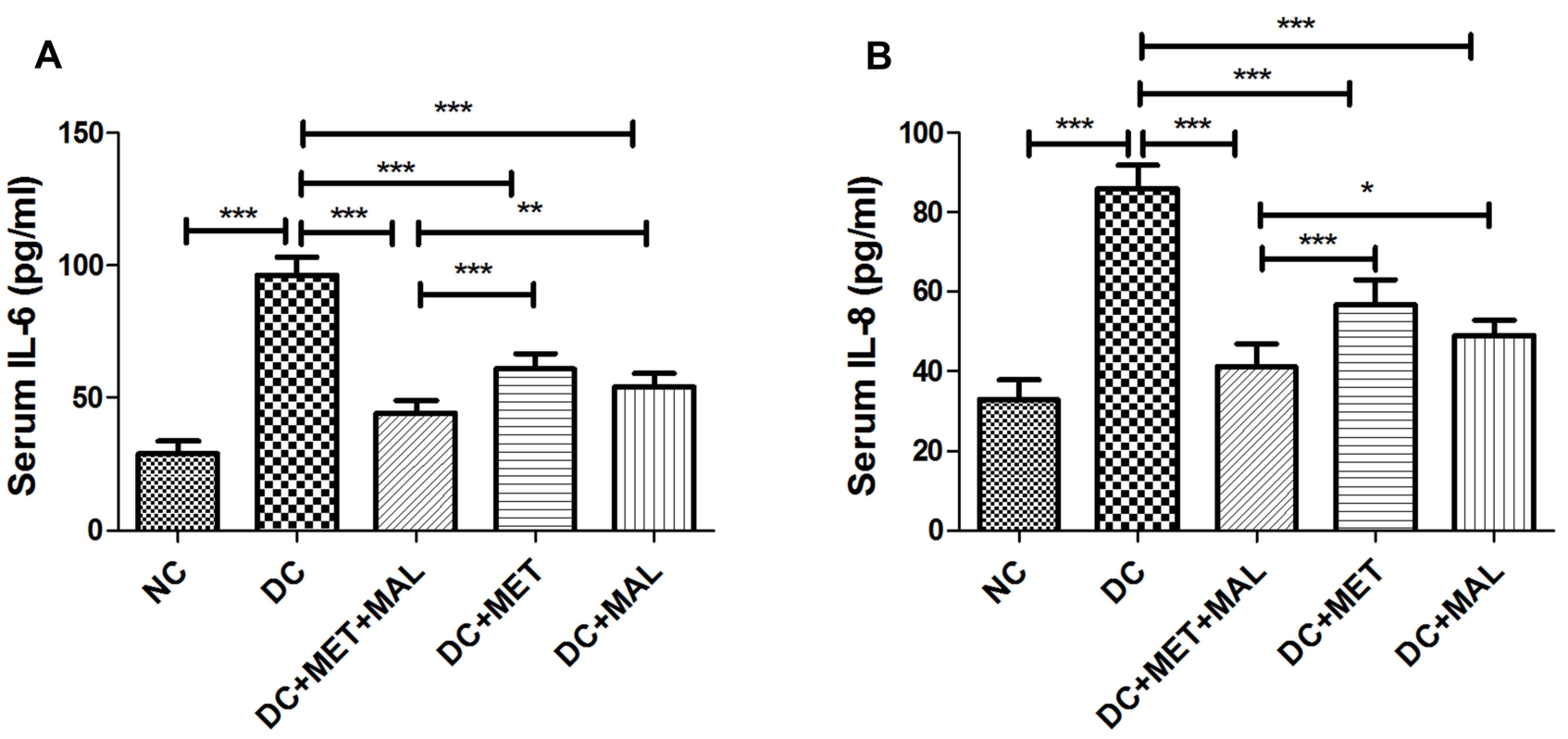

Figure 6 Effect of combined drug therapy on serum inflammatory cytokines in HFD/STZ-T2DM model rats. The serum levels of IL-6 (A) and IL-8 (B) of rats were measured by ELISA. The data were presented as mean $\pm S D,(n=8$ for all groups). $* * * P<0.001 ; * * P<0.01 ; * P<0.05$.
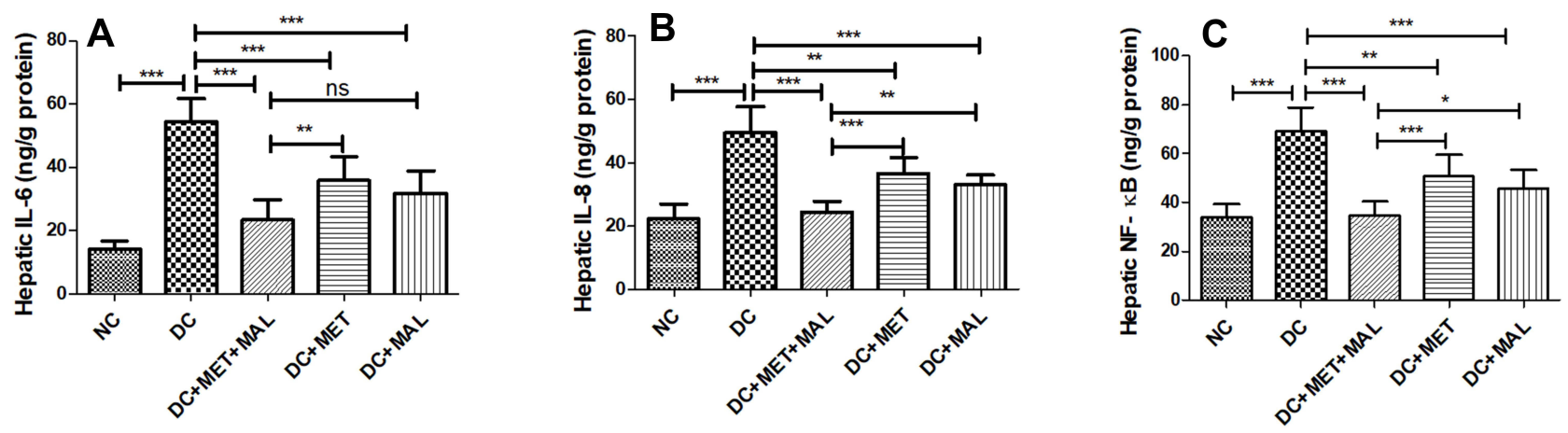

Figure 7 Effect of combined drug therapy on hepatic inflammation in HFD/STZ-T2DM model rats. The levels of IL-6 (A), IL-8 (B), and NF-kB (C) in hepatic tissue of rats were measured by ELISA. The data were presented as mean $\pm S D,(n=8$ for all groups). $* * * P<0.001 ; * * P<0.01 ; * P<0.05$.
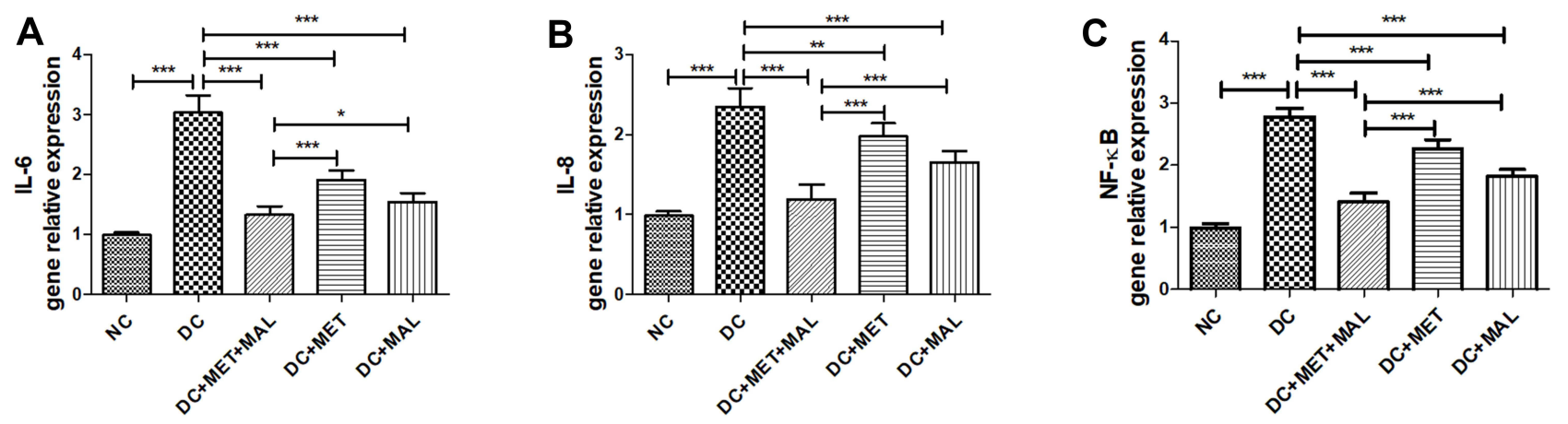

Figure 8 Effect of combined drug therapy on hepatic gene expression involved in inflammation. The relative expression of hepatic genes involved in hepatic inflammation, including IL-6 (A), IL-8 (B), and NF- $\mathrm{KB}(\mathbf{C})$. The data were presented as mean $\pm \mathrm{SD}$, $(\mathrm{n}=6$ for all groups). $* * * P<0.00 \mathrm{I} ; * * P<0.0 \mathrm{I} ; * P<0.05$. 

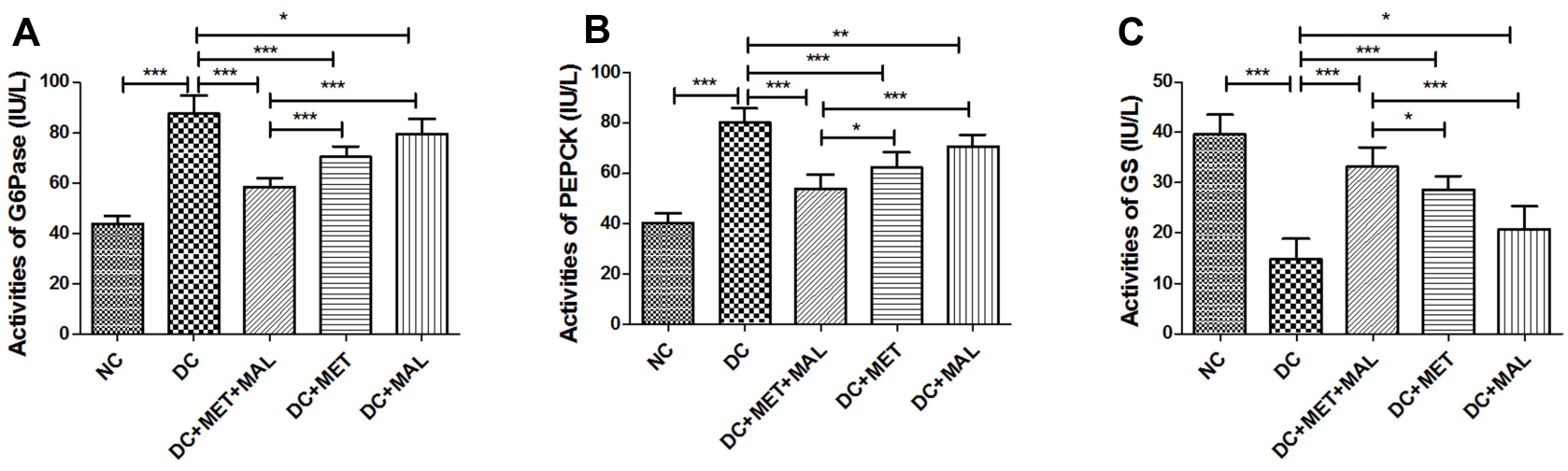

Figure 9 Effect of combined drug therapy on the activities of enzymes related to glucose metabolism. The activities of G6Pase (A), PEPCK (B), and GS (C) involved in glucose metabolism. The data were presented as mean $\pm S D,(n=8$ for all groups). $* * * P<0.001 ; * * P<0.01 ; * P<0.05$.

activity of GS in the DC group was significantly lower than those in the NC group. Treatment with MET or MAL alone increased GS activity induced by HFD/STZ. Interestingly, the increase in the $\mathrm{DC}+\mathrm{MET}+\mathrm{MAL}$ was higher than that of the MET or MAL alone.

\section{Discussion}

Increased incidence of metabolic syndromes, and T2DM due to lack of exercise and excessive intake of HFD, as a lipid metabolic disease, NAFLD is considered the most common chronic liver disease worldwide, with no approved pharmacotherapy up to date. ${ }^{29}$ Thus, it is necessary to explore novel therapy for NAFLD. The current report aimed to investigate the beneficial effects of the combination of MET and MAL in a T2DM rat model.

A T2DM rat model with NAFLD was established by HFD/STZ in the present study. Consistent with previous researches, ${ }^{30,31}$ the DC rats exhibited significant increases in fasting blood glucose, serum TC, and TG; coupled with glucose and insulin intolerance. Besides, hepatic lipid accumulation was found in the DC group, which is characteristic of NAFLD. Interestingly, the combination of MET and MAL not only reduced fasting blood glucose, serum insulin, serum lipid profiles but also alleviated insulin sensitivity. Additionally, we also observed decreased hepatic lipid accumulation in the MET + MAL group. These findings indicated that combined drug therapy could alleviate hyperglycemia, hyperlipidemia, and NAFLD in HFD/STZ-induced T2DM rats.

The previous reports have indicated that the inhibition of lipogenesis and promotion of fatty acid oxidation are important approaches for the suppression of hepatic fat accumulation. ${ }^{32-34}$ It has been reported that hepatic de novo lipogenesis is involved in the progression of NAFLD.
SREBP-1c is a transcription factor that regulates the genes involved in hepatic lipogenesis and TG synthesis, including ACC, and FAS. ${ }^{35-37}$ In the current report, the mRNA levels of SREBP-1c, ACC, and FAS were up-regulated by HFD/ STZ, resulting in elevations in hepatic TG synthesis. The combination of MET and MAL down-regulated these genes expression, leading to the declines in lipid levels. Besides, PPAR $\alpha$ could regulate several key genes involved in fatty acid oxidation and PPAR $\alpha$ activation could improve hepatic steatosis and inflammation in NAFLD. ${ }^{38} \mathrm{CPT}-1$ is an activator of PPAR $\alpha$ expression, which accelerates mitochondrial fatty acid oxidation and uptake of lipid. ${ }^{39}$ In the present study, the combination of MET and MAL upregulated PPAR $\alpha$, CPT- 1 , and LPL expression, causing an increase in fatty acid $\beta$-oxidation. These findings indicated that combined drug therapy promoted TG and fatty acid metabolism, resulting in declines in serum and liver lipid levels.

A "two-hit" process has been demonstrated that is involved in the pathogenesis of NAFLD. ${ }^{40}$ The hepatic fat accumulation evokes a series of cytotoxic events that result in a hepatic inflammatory response. ${ }^{41}$ Besides, accumulating evidence showed that inflammation is closely accompanied by lipid metabolism, is involved in the etiopathogenesis of NAFLD. ${ }^{42}$ Pro-inflammatory cytokines, such as IL-6, IL-8, and TNF- $\alpha$, have been indicated to implicate in the pathogenesis of NAFLD. ${ }^{43} \mathrm{NF}-\mathrm{kB}$ is an important regulator of liver damage and hepatic inflammatory recruitment in steatohepatitis. ${ }^{44}$ In the present study, the mRNA levels of IL-6, IL-8, and NF-kB were upregulated by $\mathrm{HFD} / \mathrm{STZ}$, resulting in the elevations in proinflammatory cytokines secretion. The combination of MET and MAL has a better effect on alleviating hepatic inflammation in HFD/STZ-induced T2DM rats. 


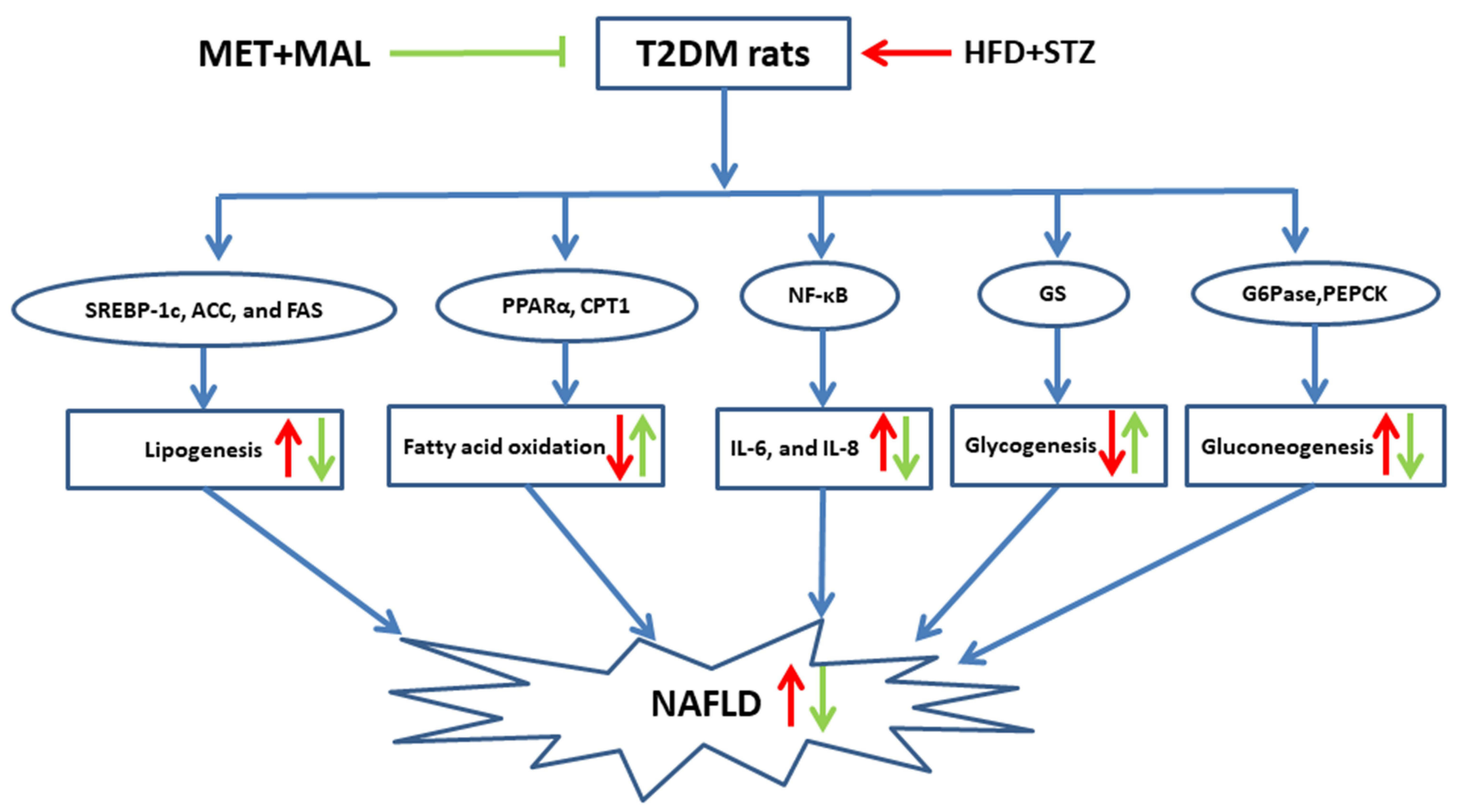

Figure 10 Recommended model for the protective mechanism of the combination of MET and MAL against NAFLD. The combination of MET and MAL prevents the progression of NAFLD by regulating lipid and glucose metabolism and inhibiting hepatic inflammation. The red arrows indicate the changes in the levels of endogenous metabolites when compared to the NC group; the green arrows indicate the changes in the levels of endogenous metabolites when compared to the DC group.

The liver is an essential place for the regulation of normal glucose metabolism. ${ }^{45}$ Insulin could suppress hepatic glycogenolysis and gluconeogenesis, and improve glucose metabolism through regulating the activity of glucose metabolismrelated enzymes such as G6Pase, PEPCK, and GS under physiological conditions. ${ }^{46}$ However, under T2DM conditions, these normal glucose metabolism processes were disordered due to insulin resistance. Our findings showed that a combination of MET and MAL could inhibit the activities of G6Pase and PEPCK, thus inhibiting hepatic gluconeogenesis. Besides, the combination of MET and MAL also could increase the activity of GS, thus promoting glycogenesis. These results indicated that combined drug therapy improved hepatic glucose metabolism via regulating the activities of glucose metabolism-related key enzymes.

Overall, for the first time, we demonstrated that a combination of MET and MAL could alleviate NAFLD via regulating lipid and glucose metabolisms, and inhibiting hepatic inflammation using HFD/STZ-induced T2DM model. The combined drug therapy presents to be more effective in inhibiting lipogenesis, promoting fatty acid oxidation, suppressing inflammatory cytokines secretion, and improving glucose metabolism than MET or MAL alone. Figure 10 indicates the role of MET $+\mathrm{MAL}$ in the alleviation of NAFLD. Therefore, this therapy could become a potential alternative therapy in the prevention and treatment of NAFLD.

\section{Disclosure}

The authors declare no conflicts of interest in this work.

\section{References}

1. GBD 2016 Disease and Injury Incidence and Prevalence Collaborators. Global, regional, and national incidence, prevalence, and years lived with disability for 328 diseases and injuries for 195 countries, 1990-2016: a systematic analysis for the Global Burden of Disease Study 2016. Lancet (London, England). 2017;390 (10100):1211-1259.

2. Mäkimattila S, Virkamäki A, Groop PH, et al. Chronic hyperglycemia impairs endothelial function and insulin sensitivity via different mechanisms in insulin-dependent diabetes mellitus. Circulation. 1996;94(6):1276-1282. doi:10.1161/01.CIR.94.6.1276

3. Bello NA, Pfeffer MA, Skali H, et al. Retinopathy and clinical outcomes in patients with type 2 diabetes mellitus, chronic kidney disease, and anemia. BMJ Open Diabetes Res Care. 2014;2(1):e000011. doi:10.1136/bmjdrc-2013-000011

4. Cheung O, Sanyal AJ. Recent advances in nonalcoholic fatty liver disease. Curr Opin Gastroenterol. 2009;25(3):230-237. doi:10.1097/ MOG.0b013e3283294a18

5. Abenavoli L, Milic N, Di Renzo L, Preveden T, Medić-Stojanoska M, De Lorenzo A. Metabolic aspects of adult patients with nonalcoholic fatty liver disease. World J Gastroenterol. 2016;22(31):7006-7016. doi:10.3748/wjg.v22.i31.7006 
6. Mantovani A, Zoppini G, Targher G, Golia G, Bonora E. Nonalcoholic fatty liver disease is independently associated with left ventricular hypertrophy in hypertensive Type 2 diabetic individuals. $J$ Endocrinol Invest. 2012;35(2):215-218. doi:10.1007/BF03345421

7. Liu D, Wong CC, Fu L, et al. Squalene epoxidase drives NAFLD-induced hepatocellular carcinoma and is a pharmaceutical target. Sci Transl Med. 2018;10:437. doi:10.1126/scitranslmed. aap9840

8. Kelley DE, McKolanis TM, Hegazi RA, Kuller LH, Kalhan SC. Fatty liver in type 2 diabetes mellitus: relation to regional adiposity, fatty acids, and insulin resistance. Am J Physiol Endocrinol Metab. 2003;285(4):E906-16. doi:10.1152/ajpendo.00117.2003

9. Dharmalingam M, Yamasandhi PG. Nonalcoholic fatty liver disease and type 2 diabetes mellitus. Indian J Endocrinol Metab. 2018;22 (3):421-428. doi:10.4103/ijem.IJEM_585_17

10. Zhang C, Lu X, Tan Y, et al. Diabetes-induced hepatic pathogenic damage, inflammation, oxidative stress, and insulin resistance was exacerbated in zinc deficient mouse model. PLoS One. 2012;7(12): e49257. doi:10.1371/journal.pone.0049257

11. Ford RJ, Fullerton MD, Pinkosky SL, et al. Metformin and salicylate synergistically activate liver AMPK, inhibit lipogenesis and improve insulin sensitivity. Biochem J. 2015;468(1):125-132. doi:10.1042/ BJ20150125

12. Pektaş MB, Sadi G, Koca HB, et al. Resveratrol ameliorates the components of hepatic inflammation and apoptosis in a rat model of streptozotocin-induced diabetes. Drug Dev Res. 2016;77(1):12-19. doi:10.1002/ddr.21287

13. Jiang C, Wang Y, Jin Q, et al. Cyclocarya paliurus triterpenoids improve diabetes-induced hepatic inflammation via the rho-kinasedependent pathway. Front Pharmacol. 2019;10:811. doi:10.3389/ fphar.2019.00811

14. Saremi A, Allison M, Ditomasso D, et al. Preliminary report: hepatic fat and inflammation in type 2 diabetes mellitus. Metab Clin Exp. 2010;59(3):430-432. doi:10.1016/j.metabol.2009.07.041

15. Foretz M, Guigas B, Bertrand L, Pollak M, Viollet B. Metformin: from mechanisms of action to therapies. Cell Metabolism. 2014;20 (6):953-966. doi:10.1016/j.cmet.2014.09.018

16. Teranishi T, Ohara T, Maeda K, et al. Effects of pioglitazone and metformin on intracellular lipid content in liver and skeletal muscle of individuals with type 2 diabetes mellitus. Met Clin Exp. 2007;56 (10):1418-1424. doi:10.1016/j.metabol.2007.06.005

17. Li YL, Li XQ, Wang YD, Shen C, Zhao CY. Metformin alleviates inflammatory response in non-alcoholic steatohepatitis by restraining signal transducer and activator of transcription 3-mediated autophagy inhibition in vitro and in vivo. Biochem Biophys Res Commun. 2019;513(1):64-72. doi:10.1016/j.bbrc.2019.03.077

18. Burton JH, Johnson M, Johnson J, Hsia DS, Greenway FL, Heiman ML. Addition of a gastrointestinal microbiome modulator to metformin improves metformin tolerance and fasting glucose levels. J Diabetes Sci Technol. 2015;9(4):808-814. doi:10.1177/ 1932296815577425

19. Zamani-Garmsiri F, Hashemnia SMR, Shabani M, Bagherieh M, Emamgholipour S, Meshkani R. Combination of metformin and genistein alleviates non-alcoholic fatty liver disease in high-fat diet-fed mice. J Nutr Biochem. 2021;87:108505. doi:10.1016/j. jnutbio.2020.108505

20. Sekar V, Mani S, Malarvizhi R, Nithya P, Vasanthi HR. Antidiabetic effect of mangiferin in combination with oral hypoglycemic agents metformin and gliclazide. Phytomed Int J Phytother Phytopharmaco. 2019;59:152901. doi:10.1016/j.phymed.2019.152901

21. Chen Z, Wang C, Pan Y, Gao X, Chen H. Hypoglycemic and hypolipidemic effects of anthocyanins extract from black soybean seed coat in high fat diet and streptozotocin-induced diabetic mice. Food Function. 2018;9(1):426-439. doi:10.1039/C7FO00983F
22. Solverson PM, Henderson TR, Debelo H, Ferruzzi MG, Baer DJ, Novotny JA. An anthocyanin-rich mixed-berry intervention may improve insulin sensitivity in a randomized trial of overweight and obese adults. Nutrients. 2019;11(12):2876. doi:10.3390/nu11122876

23. Nakano H, Wu S, Sakao K, et al. Bilberry anthocyanins ameliorate NAFLD by improving dyslipidemia and gut microbiome dysbiosis. Nutrients. 2020;12(11):3252. doi:10.3390/nu12113252

24. Bognar E, Sarszegi Z, Szabo A, et al. Antioxidant and anti-inflammatory effects in RAW264.7 macrophages of malvidin, a major red wine polyphenol. PLoS One. 2013;8(6):e65355. doi:10.1371/journal.pone.0065355

25. Wei H, Li H, Wan SP, et al. Cardioprotective effects of malvidin against isoproterenol-induced myocardial infarction in rats: a Mechanistic Study. Med Sci Monitor Int Med J Exp Clin Res. 2017;23:2007-2016. doi:10.12659/MSM.902196

26. Sakthivel KM, Kokilavani K, Kathirvelan C, Brindha D. Malvidin abrogates oxidative stress and inflammatory mediators to inhibit solid and ascitic tumor development in mice. J Environ Pathol Toxicol Oncol. 2020;39(3):247-260. doi:10.1615/JEnvironPatholToxicol Oncol.2020034430

27. Paixão J, Dinis TC, Almeida LM. Malvidin-3-glucoside protects endothelial cells up-regulating endothelial NO synthase and inhibiting peroxynitrite-induced NF-kB activation. Chem Biol Interact. 2012;199(3):192-200. doi:10.1016/j.cbi.2012.08.013

28. Wang Y, Lin J, Tian J, et al. Blueberry Malvidin-3-galactoside suppresses hepatocellular carcinoma by regulating apoptosis, proliferation, and metastasis pathways in vivo and in vitro. $J$ Agricultural Food Chem. 2019;67(2):625-636. doi:10.1021/acs.jafc.8b06209

29. Konerman MA, Jones JC, Harrison SA. Pharmacotherapy for NASH: current and emerging. J Hepatol. 2018;68(2):362-375. doi:10.1016/j. jhep.2017.10.015

30. Song D, Yin L, Wang C, Wen X. Zhenqing recipe attenuates non-alcoholic fatty liver disease by regulating the SIK1/CRTC2 signaling in experimental diabetic rats. BMC Complementary Med Therapies. 2020;20(1):27. doi:10.1186/s12906-019-2811-2

31. Srinivasan K, Viswanad B, Asrat L, Kaul CL, Ramarao P. Combination of high-fat diet-fed and low-dose streptozotocin-treated rat: a model for type 2 diabetes and pharmacological screening. Pharmacol Res. 2005;52(4):313-320. doi:10.1016/j.phrs.2005.05.004

32. Wang Y, Viscarra J, Kim SJ, Sul HS. Transcriptional regulation of hepatic lipogenesis. Nature Rev Mol Cell Biol. 2015;16(11):678-689. doi:10.1038/nrm4074

33. Sumida Y, Yoneda M. Current and future pharmacological therapies for NAFLD/NASH. $J$ Gastroenterol. 2018;53(3):362-376. doi:10.1007/s00535-017-1415-1

34. Wang J, Zhang Y, Zhuo Q, et al. TET1 promotes fatty acid oxidation and inhibits NAFLD progression by hydroxymethylation of PPAR $\alpha$ promoter. Nutr Metab. 2020;17:46. doi:10.1186/s12986-020-00466-8

35. Fang $\mathrm{K}$, $\mathrm{Wu} \mathrm{F}$, Chen $\mathrm{G}$, et al. Diosgenin ameliorates palmitic acid-induced lipid accumulation via AMPK/ACC/CPT-1A and SREBP-1c/FAS signaling pathways in LO2 cells. BMC Complement Altern Med. 2019;19(1):255. doi:10.1186/s12906-019-2671-9

36. Goedeke L, Bates J, Vatner DF, et al. Acetyl-CoA carboxylase inhibition reverses NAFLD and hepatic insulin resistance but promotes hypertriglyceridemia in rodents. Hepatology (Baltimore, Md. 2018;68(6):2197-2211. doi:10.1002/hep.30097

37. Watanabe M, Houten SM, Wang L, et al. Bile acids lower triglyceride levels via a pathway involving FXR, SHP, and SREBP-1c. J Clin Invest. 2004;113(10):1408-1418. doi:10.1172/JCI21025

38. Pawlak M, Lefebvre P, Staels B. Molecular mechanism of PPAR $\alpha$ action and its impact on lipid metabolism, inflammation and fibrosis in non-alcoholic fatty liver disease. J Hepatol. 2015;62(3):720-733. doi:10.1016/j.jhep.2014.10.039 
39. Kersten S, Seydoux J, Peters JM, Gonzalez FJ, Desvergne B, Wahli W. Peroxisome proliferator-activated receptor alpha mediates the adaptive response to fasting. $J$ Clin Invest. 1999;103 (11):1489-1498. doi:10.1172/JCI6223

40. Day CP, James OF. Steatohepatitis: a tale of two "hits"? Gastroenterology. 1998;114(4):842-845. doi:10.1016/S00165085(98)70599-2

41. Manne V, Handa P, Kowdley KV. Pathophysiology of nonalcoholic fatty liver disease/nonalcoholic steatohepatitis. Clin Liver Dis. 2018;22(1):23-37. doi:10.1016/j.cld.2017.08.007

42. Cobbina E, Akhlaghi F. Non-alcoholic fatty liver disease (NAFLD) pathogenesis, classification, and effect on drug metabolizing enzymes and transporters. Drug Metab Rev. 2017;49(2):197-211. doi:10.1080/ 03602532.2017 .1293683
43. Tilg H, Moschen AR. Evolution of inflammation in nonalcoholic fatty liver disease: the multiple parallel hits hypothesis. Hepatology (Baltimore, Md. 2010;52(5):1836-1846. doi:10.1002/hep.24001

44. Boden G, She P, Mozzoli M, et al. Free fatty acids produce insulin resistance and activate the proinflammatory nuclear factor-kappaB pathway in rat liver. Diabetes. 2005;54(12):3458-3465. doi:10.2337/diabetes.54.12.3458

45. Han P, Cui Q, Lu W, et al. Hepatocyte growth factor plays a dual role in tendon-derived stem cell proliferation, migration, and differentiation. $\quad J$ Cell Physiol. 2019;234(10):17382-17391. doi: $10.1002 /$ jcp. 28360

46. Klover PJ, Mooney RA. Hepatocytes: critical for glucose homeostasis. Int $J$ Biochem Cell Biol. 2004;36(5):753-758. doi:10.1016/j.biocel.2003.10.002

\section{Publish your work in this journal}

Drug Design, Development and Therapy is an international, peerreviewed open-access journal that spans the spectrum of drug design and development through to clinical applications. Clinical outcomes, patient safety, and programs for the development and effective, safe, and sustained use of medicines are a feature of the journal, which has also been accepted for indexing on PubMed Central. The manuscript management system is completely online and includes a very quick and fair peer-review system, which is all easy to use. Visit http://www. dovepress.com/testimonials.php to read real quotes from published authors. 